\title{
The Influence of Communication on Knowledge Integration in CFTs: A Case of Chinese Mobile Applications Enterprise
}

\author{
Yixuan Wang \\ Assistant Professor, Nagoya University, \\ Furo-cho, Chikusa-ku, Nagoya 464-8601, Japan
}

Received: May 6, 2018 Accepted: June 5, 2018 Online published: June 27, 2018

doi:10.5296/ijhrs.v8i3.13327ＵRL: https://doi.org/10.5296/ijhrs.v8i3.13327

\begin{abstract}
We examine the relationship between communication and knowledge integration (KI) in the context of a cross-functional teams (CFTs). Although communication is one of the most important processes for integrating knowledge in CFTs, little research examines the relationship between them. We adopt both qualitative and quantitative research methods in a Chinese mobile applications enterprise. We find that team communication plays a key role in the KI of CFTs. Team members should have sufficient communication with each other for cross-functional activities. Also, communication network structure in an enterprise can influence KI. Thus, enterprises need to select suitable team members before the project begins for effective KI in CFTs.
\end{abstract}

Keywords: knowledge integration, cross-functional teams, the degree of centrality, communication time

\section{Introduction}

Nowadays, the Mobile Application Software Industry (MASI) is growing fast, and companies are facing intense competition in the Chinese market. Many companies in MASI aim at producing innovative products by cross-functional teams (CFTs). The CFT members are from different departments in companies; they have relative expertise in solving companies' proposed innovation problems. However, their professional backgrounds, departmental cultures, and languages are different (Dougherty, 1992). Thus they always have difficulties in communication and knowledge integration (KI).

Berggren, Bergek, Bengtsson, \& Söderlund (2011) advocated that KI is a process of 
collaborative and purposeful combination of complementary knowledge between individuals, teams, and firms. This process usually involves significant elements of new knowledge generation. In this paper, we will discuss the relationship between communication and $\mathrm{KI}$ in CFTs. Exploring KI in CFTs is important because firms are increasingly relying on this form of organization (Huang \& Newell, 2003). In MASI, forming KI for integrating multi-disciplinary knowledge is central to develop application software. Previous researchers have discussed many influential factors of KI in CFTs, such as social capital (Newell, Tansley, \& Huang 2004; Robert, Dennis \& Ahuja, 2008), common knowledge (Grant, 1996; Huang \& Newell, 2003), knowledge relatedness (Nesta \& Saviotti, 2006), past integration experience (Huang \& Newell, 2003), and formal intervention (Okhuysen \& Eisenhardt, 2002). However, research the relationship between communication and $\mathrm{KI}$ is rare. We address the following questions:

1. Can team communication positively affect KI in CFTs? If so, Why?

2. Can team members' communication network inside the company positively influence the $\mathrm{KI}$ in CFTs? Why?

To find answers to those questions, we flew to a site of a Chinese Mobile Application Company to collect communication network and team performance data.

\section{Theoretical Background}

\subsection{Knowledge and Cross-Functional Teams in Software Development}

Rus \& Lindvall (2002) illustrated that technical knowledge and application domain knowledge are two types of knowledge in software development process. Technical knowledge is the one about design, programming, and software processes. Business application domain knowledge refers to knowledge about customers' business processes, business rules, activities, stakeholder needs, and the customers' business objectives for the software (Tiwana, 2004). For instance, in MPAI, "programmers" know how to program software and "user interface designers" know how to design interface of the software. Thus, they possess the technical knowledge. On the other side, "marketers" and "operators" possess the application domain knowledge. Marketers know how to promote for attracting the customers and operators know how to analyze user data for retaining customers. In short, application domain knowledge is related to the customers' and end-users' needs while technical knowledge is related to the technical solution of the needs, respectively. Thus, it is vital to integrate both technical knowledge and application domain knowledge for software development by CFTs.

For integrating technical knowledge and application knowledge, many organizations have created teams in which members of various functions work together to bring their respective skills and perspectives to a common work output (Wynn \& Novick, 1995). The formation of CFTs aims to solve the problem what individual function/department cannot achieve. The CFT activities are usually related to new product development (Clark \& Fujimoto, 1991), import of the new system (Newell, Tansley, \& Huang, 2004), exploration and innovation (Kleinsmann, Buijs \& Valkenburg, 2010), boundary spanning (Ancona and Caldwell, 1992). 
However, some literature pointed out that the difficulties of KI might appear, when the enterprises increasingly depend on this type of organizational form (e.g., Huang \& Newll, 2003; Majchrzak, More, \& Faraj, 2012).

Firstly, since team members in CFTs are from different departments of the enterprises, each team member represents a different "thought world." Thus each member understands the problems, critical elements, and steps in solving the problem differently from each other member (Dougherty, 1992). This heterogeneity of knowledge domain could facilitate "creative abrasion" (Iansiti, 1995), especially, the creative abrasion between the members who possess technical knowledge and application domain knowledge is more obvious compared with homogeneous knowledge. However, difficulties of understanding of each other's laws of thought might cause a negative effect to team performance as well. Secondly, Denison, Hart, \& Kahn (1996) indicated that CFTs would be under pressure of achieving the goals of the projects, because, there is a time limitation for the mission of the project, and team members have to balance both daily work and project mission in the meantime. Thus, the balance of time, cost and goals are the problems that often puzzles the CFT members. Thirdly, team members possess the social identity of own department (Denison, Hart, \& Kahn, 1996). Occasionally, team members excessively insist functional social identity or culture and start to become sectionalism that negatively affects the team performance. The CFT is extremely hard to implement communication and KI effectively in above three aspects. Also, the research about KI across organizational boundaries is still limited (Ruan, Ochieng, Price, \& Egbu, 2012). Thus, our study targets the relationship between communication and KI in this type of special organizational form.

\subsection{Knowledge Integration and Communication}

Grant (1996) systematically constructed the theory of KI. He admired that advances in knowledge require combining specializations of many individuals within the organization for sustaining competitive advantages. The primary role of an organization is to integrate specialized knowledge. Although there are various arguments on the concept of KI, most researchers agree that $\mathrm{KI}$ is a process that combines complementary knowledge (Tell, 2011). The KI process usually involves sharing, combining and creating the knowledge (Okhuysen $\&$ Eisenhardt, 2002). This paper mainly focuses on KI on the team level and the influential factors as shown below.

Common knowledge is one of the most discussed influential factors in KI field. As far back as Grant (1996), he admired the level of KI efficiency depends on the extent to which common knowledge exists among participants. To form a common knowledge in a multi-disciplinary team, researchers also suggested that sharing specialists' "deep knowledge" and developing extensive deep-knowledge dialogue could break knowledge boundaries and promote KI (e.g., Majchrzak, More, \& Faraj, 2012). Another influential factor is social capital. Robert, Dennis, \& Ahuja (2008) studied the performances appearing in 46 teams. The result shows that all three dimensions of their social capital (structural, relational, and cognitive) impact on KI. The structural and cognitive capital was more important to KI when teams communicated through lean digital networks than when they communicated 
face-to-face; relational capital directly impacted KI equally regardless of the communication media used by the team. Huang \& Newell (2003) also investigated CFTs in 4 different companies and pointed out the positive relationship between social capital and KI. Also, their case study indicated that enterprises' past integration experience also affected KI and could make integration process much easier. On the other hand, Okhuysen \& Eisenhardt (2002) discussed effects by using three simple formal interventions (information sharing, questioning others and managing time). They significantly changed their work process and improved the KI. In particular, groups in questioning others and managing time conditions exhibited greater KI than groups purely in the information sharing, and control conditions did.

In sum, there are various influential factors on KI; however little research has deeply discussed the relationship between communication and KI. Huang \& Newell (2003) mentioned frequent communication is a need in CFTs, and Kleinsmann, Buijs, \& Valkenburg (2010) also pointed out sufficient team communication is necessary for New product development teams (NPD). Thus, communication could play a key role in integrating team members' knowledge, about which we still know rarely. Here, we will study how and why the communication can affect the KI.

\section{Research Framework and Hypothesis}

We propose that two dimensions of communication can affect KI in CFTs. As shown in Fig.1, the one dimension is team communication, and the other is communication network structure. The role of team communication means that team members communicate with each other within CFTs. Since the team communication plays a key role in CFTs (Huang \& Newell, 2003; Kleinsmann, Buijs \& Valkenburg 2010), here, we temporally suppose that team communication will positively affect KI.

Two Dimensions of Communication

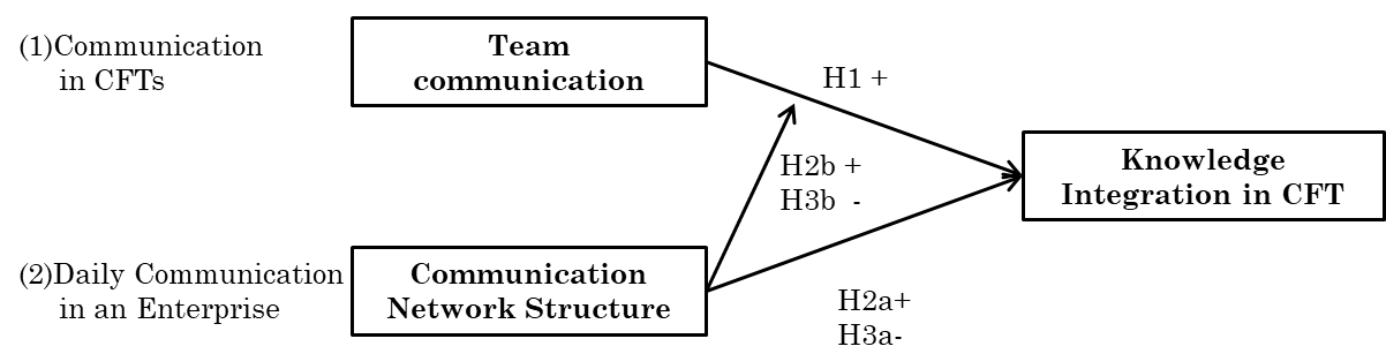

Figure 1. The Framework of the Relationship between Communication and Knowledge Integration

Hypothesis 1 (H1): Frequent team communication will lead to an increase in KI in CFTs

Communication network structure is constructed by the employee's communication which is related to daily work in the enterprise. We may find a reliable employee who has more connection with others, he or she has more information and power comparing with the other people who have not. Thus, this kind of employee who has many connections with others may more promote team performance. We assumed that communication network structure 
has a direct positive impact on $\mathrm{KI}$.

The final assumption is that communication network structure also may indirectly promote team communication. As we mentioned above, an employee who has many connections with others, they may be good at communication, because, he or she has more experience compare with the one who has fewer connections with others. Thus communication network structure may facilitate the relationship between communication and KI. However, higher differences in network connections between team members also may cause direct or indirect a negative impact on KI, since there will be a large knowledge gap between team members.

Hypothesis 2a (H2a): A team member's network connections in an enterprise will positively influence KI in CFTs.

Hypothesis 2b (H2b): A team member's network connections in an enterprise will positively facilitate the relationship between communication and $\mathrm{KI}$ as a moderator.

Hypothesis 3a (H3a): Higher differences of network connections between team members will negatively influence KI in CFTs.

Hypothesis 3a (H3b): Higher differences of network connections between team members will negatively facilitate the relationship between communication and $\mathrm{KI}$ as a moderator.

\section{Methodology}

To focus on examining the relationship between communication and KI in CFTs, we followed the multi-strategy research by Brayman (2006). We are carrying on our study in both quantitative and qualitative aspects. There are two reasons why multi-strategy research method is suitable for our research. First, since we consider the impact from two dimensions of communication (Fig. 1), there is more than one source of data to use for the solution of the research problems (Tashakkori \& Teddlie, 2003). We can have one data set for the communication and performance within the team, and have another one for the daily communication network within an enterprise. Second, qualitative evidence can explain some of the relationships uncovered through an analysis of quantitative data (Bryman, 2006) and solve "how" and "why" questions (Yin, 2017). Thus, the qualitative method could complement the quantitative method. All the research data was collected by "CHANGE" which is a Chinese mobile phone application company. Our some interview data is similar to the Wang and Jiang (2018). However, the interview data in this study is more focus on team communication, and their study did not use the quantitative data of communication network.

We did field research and collected network and interview data from July 2015 to September 2015. About one and a half months on-site observation is carried out by the author. Such an observation enabled researchers to understand organization's background, organizational structure, organizational culture and sub-culture, technology and business processes (Huang $\&$ Newell, 2003). The process of collecting the network and interview data is as follows.

\subsection{Data Collection: Communication Network Structure Data within the Enterprise}

To grasp the daily communication in CHANGE we collected two weeks face to face 


\section{Ml Macrothink}

International Journal of Human Resource Studies

ISSN 2162-3058

2018, Vol. 8, No. 3

communication data (10 working days) from 59 employees (whole members of the company) during the on-site observation period. Each employee of CHANGE had filled the survey questions every day. As long as an employee started a working conversation, a record would remain. These communication records included both employees' name and period of the conversation. This survey could help us to know the connections between organization members in CHANGE. Consequently, we draw the entire communication network structure as shown in Fig. 2. Nodes indicate organization members and ties (lines) indicate communication between employees in CHANGE's daily work. Communication network structure data will be used to calculate independent variables- higher value in the degree of centrality and the differences in the degree of centrality respectively in a later section.

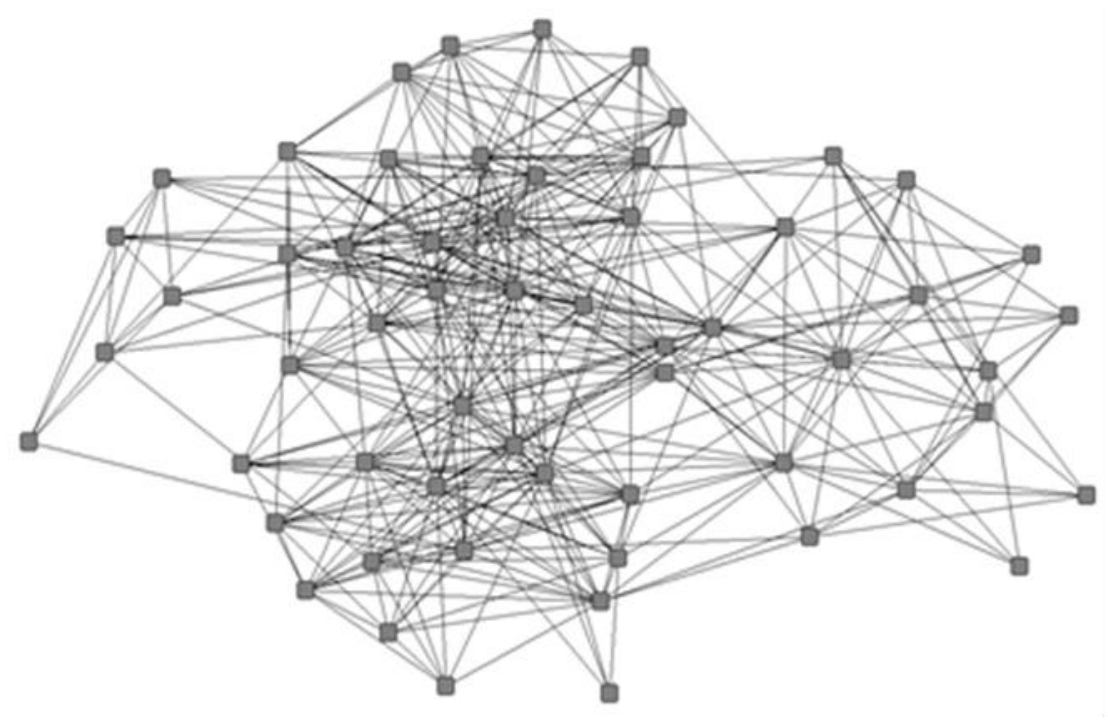

Figure 2. Communication Network Structure in "CHANGE"

\subsection{Data Collection: The Result of Knowledge Integration}

A project called "Anyone could be a product manager" in CHANGE was carried out from September 2015 to November 2015, from which we obtained the team performance data. 32 employees joined the project, in which two employees formed a team. Thus, there are 16 teams for this project. CHANGE randomly formed Each CFT by a lucky draw with different genders (4 departments join the project, i.e., the departments of market, content, and operation, Technology and Design). The project aimed to find out product problems in CHANGE and provide improvement advice from team members within three days for each team. The product manager and the chief technology officer (CTO) of CHANGE immediately evaluated suggestions or solutions of each team and gave the accumulated scores for them. In this research, accumulated scores of each team is a proxy variable for the result of KI.

\subsection{Data Collection: Interview Data}

To complement quantitative result, we interviewed all 32 team members who attended the project. Our interviews focused on understanding how and why communication affects KI. 


\section{Ml Macrothink}

International Journal of Human Resource Studies

ISSN 2162-3058

2018, Vol. 8, No. 3

The questions were: "How did they communicate with each other?", "How did they combine the solutions?" and "Why did some teams perform well or not?". We also interviewed 1 product manager and $1 \mathrm{CTO}$ who were responsible for evaluating the performance of teams to meet the requirement of the whole project. On average, each respondent cost 1.5 hours for the interview.

\subsection{The Dependent Variable}

The accumulated scores by the evaluation of "CHANGE" measure the result of knowledge integration. We used team evaluation scores as a proxy variable since $\mathrm{KI}$ is based on the suggestions or solutions of teams in our case. Their evaluation system for each advice has five ranks (A to E): rank A is 9 scores, rank B is 5 scores, rank $C$ is 3 scores, rank D is 1 score and rank $\mathrm{E}$ is 0 score respectively. If a team got one rank $\mathrm{A}$, two rank $\mathrm{C}$, and one rank $\mathrm{E}$, the performance of the team should be 15 scores $(9+3+3+0=15)$. It is better that we obey the enterprise evaluation system as the performance scale because only the enterprise itself can measure and judge the value of solutions and suggestions.

\subsection{Independent Variables}

There are two scales of independent variables. One is the Team communication, and the other one is communication network structure.

Communication time (minutes) measures team communication. There is some argument for communication time in KI field. Grant (1996) believed that when an organization integrates its knowledge, communication should be less for the efficiency of integration. However, Huang \& Newell (2003) and Kleinsmann, Buijs, \& Valkenburg (2010) argued that the CFT members need frequent communication to raise the efficiency of KI.

A higher value in the degree of centrality and the differences in the degree of centrality measure communication network structure. The degree of centrality indicates how many ties nodes have (here is each employee in CHANGE). A high degree of centrality for an individual node manifests that it has many links compared to other nodes (Freeman, Roeder, \& Mulholland, 1979). We pick up a higher value in the degree of centrality as an independent variable. We proposed that a team member who is with a higher value in the degree of centrality would play more roles in team performance. The differences in the degree of centrality indicate whether the similar or different value in the degree of centrality in a team. We proposed that a large difference in the degree of centrality in a team could negatively affect KI. We used UCINET software (Borgatti, Everett, \& Freeman, 2002) to analyze these variables.

\subsection{Control Variables}

Although previous research discussed team size, genders and time constraint may affect KI differently (e.g., Robert, Dennis, \& Ahuja, 2008), in this research all those factors are the same. The differences in work experience and contact in daily work are control variables. Work experiences are quite different between employees, and it may strongly affect KI. On the other hand, some team members who worked longer period together in daily work may 


\section{Macrothink}

also affect KI.

\section{Results and Discussion}

\subsection{Results}

Descriptive statistics and the results of ordinary least squares (OLS) are in table 1 and 2, respectively. Table 1 shows fewer correlations among all the independent variables. Model 1 , Model 2, and Model 3 test dependent variables, dependent variables with control variables, and dependent variables with control variables and interaction, respectively. Model 3 contains an interaction between the higher value in the degree of centrality and communication time, the interaction between the differences in degree of centrality and communication time, an interaction between the differences in work experience. Model 3 is the most reliable model since the variables are complete and adjusted $R^{2}$ is the highest value $\left(R^{2}=0.812\right)$ in all three models. The communication time is positive and significant in all three models. Thus, this result supports $\mathrm{H} 1$. The differences in the degree of centrality, the interaction between a higher value in the degree of centrality and communication time, and the interaction between the differences in degree of centrality and communication time are significantly negative, positive, and negative, respectively.

Table 1. Descriptive Statistics

\begin{tabular}{|c|c|c|c|c|c|c|c|}
\hline Variable & Mean & S.D. & 1 & 2 & 3 & 4 & 5 \\
\hline 1. Outcome of knowledge integration & 10.813 & 6.231 & & & & & \\
\hline 2. Communication time & 17.125 & 17.580 & $0.572^{*}$ & & & & \\
\hline 3. Higher value in degree of centrality & 18.438 & 5.075 & -0.248 & -0.588 & & & \\
\hline 4. The differences in degree of centrality & 6.813 & 4.535 & -0.221 & -0.690 & 0.436 & & \\
\hline 5. The differences in work experience & 2.813 & 2.664 & 0.235 & -0.200 & 0.401 & 0.334 & \\
\hline 6. Contact in daily work & 0.125 & 0.342 & 0.482 & 0.008 & 0.390 & 0.113 & 0.394 \\
\hline $\mathrm{N}=16^{*}: \mathrm{p}<0.05 ; * *: \mathrm{p}<0.01$ & & & & & & & \\
\hline
\end{tabular}

The effect of higher value in the degree of centrality is not significant in all of the three models. However, the effect of the interaction between the higher value in the degree of centrality and communication is tested in model 3. Thus, this result supports $\mathrm{H} 2 \mathrm{~b}$ and rejects $\mathrm{H} 2 \mathrm{a}$. The effect of the differences of degree of centrality is not tested in model 1 and model 2. However, it is tested in model 3. Also, the effect of an interaction between the differences in degree of centrality and communication time is also tested. Thus this result supports $\mathrm{H} 3 \mathrm{a}$ and H3b. 


\section{Macrothink \\ International Journal of Human Resource Studies \\ ISSN 2162-3058 \\ 2018, Vol. 8, No. 3}

Table 2. The Effects of Communication on Knowledge Integration

\begin{tabular}{lccc}
\hline Variable & Model 1 & Model 2 & Model 3 \\
\hline Communication time & $0.74^{* *}$ & $0.55^{+}$ & $1.70^{* *}$ \\
Higher value in degree of centrality & 0.32 & -0.11 & 0.32 \\
The differences in degree of centrality & 0.31 & -0.20 & $-0.40^{+}$ \\
The differences in work experience & & 0.31 & $0.49^{*}$ \\
Contact in daily work & & 0.38 & -0.22 \\
Higher value in degree of centrality $\times$ Communication time & & & $1.18^{* *}$ \\
The differences in degree of centrality $\times$ Communication time & & & $-0.54^{* *}$ \\
The differences in work experience $\times$ Communication time & & & $0.83^{*}$ \\
Contact in daily work $\times$ Communication time & & & 0.57 \\
Model fit & & & \\
R-squared & 0.412 & 0.640 & 0.925 \\
Adj. R-squared & 0.265 & 0.459 & 0.812 \\
Model improvement $F-$ test & $2.801^{* *}$ & $3.549^{* *}$ & $8.199^{* *}$ \\
\hline$+: p<0.10 ; *$ p $<0.05 ; * * p<0.01$ & & &
\end{tabular}

\subsection{Discussion}

The result shows that longer communication time is most recommended for KI when team members integrate knowledge in CFTs. This result leads to a question raising here: Why can team communication time positively affects KI in CFTs? To answer this question, we should define the differences related to the communication time between teams. We observed three differences in interview data.

The first difference is commitment problem. For example, the communication time of team 9 was extremely short. Team members communicated only lasting 5 minutes during the project. A team member was against the project. "Why should I join this project? Does it mean we should hold anyone could be a programmer next time?" He expressed his feeling. He also thought that this kind of project should be voluntary, not be forced. Commitment problem resulted in team 9 lack of communication, inefficient KI and the poor result (only 1 score). On the contrary, the communication time of team 8 was longer compare with most of the team. During the interview, both team members liked the project and a member said: "This project is great." "You know...we have so many problems with our product, but nobody says it." "Anyone could be a product manager gave us a place that we can express our ideas" As a result, team 8 had enough communication (60 minutes), which resulted in a good KI and a satisfying result (16 scores).

The second difference is schedule problem. The communication time of team 10 was the least in all teams. Team 10 communicated 0 minutes in the project. A member who is from marketing department was extremely busy during the project. "I was negotiating with clients at that time. I wanted to discuss with a partner, but I did not have time. To be honest, I contributed nothing, and I felt guilty....." he said. As are result KI is insufficient, and they 
got 0 scores.

The third difference is the way of KI. Some teams did project separately and combine all the solutions at last. For instance, team members in team 7 (2 minutes 2 scores) didn't share their suggestions or discuss their ideas sufficiently, they only put all the solutions together and submitted it. On the contrary, team 3 shared their ideas and discussed which suggestions are good or bad (taking 36 minutes and getting13 scores). Thus, short communication and long communication are different in the way of KI, and it will affect the quality of KI significantly.

In sum, teams with longer communication time have obvious advantages in the issues of commitment, schedule and the way of KI comparing with teams using less communication time. These three differences are the reasons of why communication time can positively affect KI. Grant (1996) proposed minimizing the need for communication, which was opposite to our study. Our results assisted the suggestions of Huang \& Newell (2003) and Kleinsmann, Buijs \& Valkenburg (2010). Certainly, there is not a need to take too much time if the communication is less necessary. However, since cross-functional projects are non-routine tasks (Huang \& Newell, 2003), a sufficient communication might necessary. Note that we do not recommend the way of KI that is "work separately before combining."

The result shows that higher value in the degree of centrality facilitates the relationship between the communication and KI. It implies that a member who is with a higher value in the degree of centrality can make KI efficiently. During the interview, we confirmed that a member who is with a higher value in the degree of centrality could play a role of a leader. They may not be officially ordered to be a leader. However, what they did in their work could be very active in showing strong leadership. For instance, a team 4 member said "My partner was professional, and his logic was awesome. He can point out the crucial point of our product......Yes! He combined our work efficiently. "We found that team 1 and team 5 also had an "integrator" who was with a higher value in the degree of centrality. The integrator induced communication, combined ideas and made cooperation better. Thus integrator facilitates the relationship between the communication and KI.

Another result shows that the differences in degree of centrality might negatively impact on $\mathrm{KI}$ in CFTs and it might minify the relationship between communication and KI as well. This case indicates that if the gap of the degree of centrality is large among team members, the result of KI might be poor. However, if both team members are of high value in the degree of centrality or both team members are of low value in the degree of centrality, the result of KI might be good. A member of the team 12 with a large gap (the differences are 12 ties) said: "I did not feel like to communicate a lot...He talked little...He was not so professional..." and another team member said: "it was not easy to start the conversation... You know, she is in manager position..." In this team, the member who was with a higher degree of centrality did not feel like to share knowledge with another member. She did not think her partner is capable for the project. The team member who was with a lower degree of centrality was usually afraid to talk with his partner because he was lack of confidence in his ability. The employee who was with a high value in the degree of the centrality might have more information, knowledge and power compare with others. Both members had high value in the 
degree of centrality in team 4 (the differences are 2 ties). They felt efficient and cooperated comfortably with each other. We found that both members were with low value in the degree of centrality in team 3 (the differences are 2 ties). However, they performed well on the KI (16 scores). The interview data indicated that although both of them had little chance to give suggestions on daily work, they hurled themselves into the project. They felt excited and motivated during the project. Thus they took much more communication time compare with others. In sum, if the gap of the knowledge level between members is too large, an asymmetric power will occur, and it will negatively impact on KI eventually.

\section{Conclusion}

In this paper, we discussed how and why the communication affects $\mathrm{KI}$ in detail. The results demonstrate that communication time positively impacts on KI in CFTs; a team member who is with a higher value in the degree of centrality would strengthen the relationship between communication and KI; the differences in degree of centrality between team members has a negative impact on KI. This empirical evidence show that communication plays a key role when CFTs integrate the knowledge of team members.

\subsection{Contribution}

There are several theoretical contributions in this paper. (1) This paper pointed out that team communication is important to KI in CFTs. Sufficient communication is also recommended among team members. (2) Communication network structure can influence KI. Thus the higher level of organization units (enterprises) affects the KI at lower levels of organization units (KI). Even if an enterprise produces a temporal organization for KI, the temporal organization would be strongly affected by the communication network structure of the enterprise.

There are also several empirical contributions in this paper. (1) The enterprise should set specific rule for KI. In the project of "anyone could be a project manager" the enterprise did not tell them how to combine the knowledge and didn't set the communication time. If the enterprise does not set the specific rules as a time constraint for KI, the result will be unrespectable. (2) An enterprise should not put the members who have a large gap in the degree of centrality to form a team, because there is an asymmetric power between them and the result might be poor.

\subsection{Limitation and Future Directions}

The limitations of this paper are: (1) More team numbers and bigger team size are desired in a future study. (2) The sample size of teams in quantitative part is small. However, the data in this paper is really rare and difficult to get. The qualitative data also complement the problem of lack of sample problem in quantitative research. (3) We need more data collection and more interviews to study it in future since this is an analysis by using the data collected only from one company. In the future work, we will conduct the comparative case study for the deeper understanding of the mechanism of communication and KI. 


\section{References}

Berggren, C. (Ed.). (2011). Knowledge integration and innovation: Critical challenges facing international technology-based firms. Oxford University Press. https://doi.org/10.1093/acprof:oso/9780199693924.001.0001

Borgatti, S. P., Everett, M. G., \& Freeman, L. C. (2002). Ucinet for Windows: Software for social network analysis. Analytic Technologies, Natick, MA.

Bryman, A. (2006). Integrating quantitative and qualitative research: how is it done?. Qualitative research, 6(1), 97-113. https://doi.org/10.1177/1468794106058877

Clark, K. B., \& Fujimoto, T. (1991). Product Development Performance, Boston, MA: Harvard Business School Press.

Denison, D. R., Hart, S. L., \& Kahn, J. A. (1996). From chimneys to cross-functional teams: Developing and validating a diagnostic model. Academy of management journal, 39(4), 1005-1023.

Dougherty, D. (1992). Interpretive barriers to successful product innovation in large firms. Organization Science, 3(2), 179-202. https://doi.org/10.1287/orsc.3.2.179

Freeman, L. C., Roeder, D., \& Mulholland, R. R. (1979). Centrality in social networks: II.

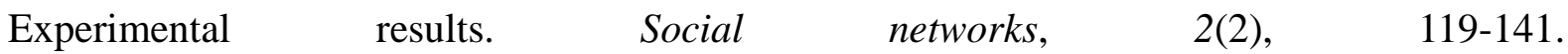
https://doi.org/10.1016/0378-8733(79)90002-9

Grant, R. M. (1996). Prospering in dynamically-competitive environments: Organizational capability as knowledge integration. Organization Science, 7(4), pp.375-387. https://doi.org/10.1287/orsc.7.4.375

Huang, J. C., \& Newell, S. (2003). Knowledge integration processes and dynamics within the context of cross-functional projects. International journal of project management, 21(3), 167-176. https://doi.org/10.1016/S0263-7863(02)00091-1

Iansiti, M. (1995). Shooting the rapids: Managing product development in turbulent environments. California Management Review, 38(1), 37-58. https://doi.org/10.2307/41165820

Kleinsmann, M., Buijs, J., \& Valkenburg, R. (2010). Understanding the complexity of knowledge integration in collaborative new product development teams: A case study. Journal of Engineering and Technology Management, 27(1-2), 20-32. https://doi.org/10.1016/j.jengtecman.2010.03.003

Majchrzak, A., More, P. H., \& Faraj, S. (2012). Transcending knowledge differences in cross-functional teams. Organization Science, 23(4), 951-970. https://doi.org/10.1287/orsc.1110.0677

Nesta, L., \& Saviotti, P. P. (2006). Firm knowledge and market value in biotechnology. Industrial and Corporate Change, 15(4), 625-652. https://doi.org/10.1093/icc/dt1007 


\section{Macrothink}

International Journal of Human Resource Studies

ISSN 2162-3058 2018, Vol. 8, No. 3

Newell, S., Tansley, C., \& Huang, J. (2004). Social capital and knowledge integration in an ERP project team: the importance of bridging and bonding. British Journal of Management, 15(S1). https://doi.org/10.1111/j.1467-8551.2004.00405.x

Okhuysen, G. A., \& Eisenhardt, K. M. (2002). Integrating knowledge in groups: How formal interventions enable flexibility. Organization Science, 13(4), 370-386. https://doi.org/10.1287/orsc.13.4.370.2947

Robert Jr, L. P., Dennis, A. R., \& Ahuja, M. K. (2008). Social capital and knowledge integration in digitally enabled teams. Information Systems Research, 19(3), 314-334. https://doi.org/10.1287/isre.1080.0177

Ruan, X., Ochieng, E. G., Price, A. D., \& Egbu, C. O. (2012). Knowledge integration process in construction projects: a social network analysis approach to compare competitive and collaborative working. Construction Management and Economics, 30(1), 5-19. https://doi.org/10.1080/01446193.2011.654127

Rus, I., \& Lindvall, M. (2002). Knowledge management in software engineering. IEEE Software, 19(3), 26. https://doi.org/10.1109/MS.2002.1003450

Tashakkori, A., \& Teddlie, C. (Eds.). (2010). Sage handbook of mixed methods in social \& behavioral research. London: Sage. https://doi.org/10.4135/9781506335193

Tell, F. (2011). Knowledge integration and innovation: a survey of the field. Knowledge Integration and Innovation: Critical challenges facing international technology-based firms, 25,69 .

Tiwana, A. (2004). An empirical study of the effect of knowledge integration on software development performance. Information and Software Technology, 46(13), 899-906. https://doi.org/10.1016/j.infsof.2004.03.006

Wang, Y., \& Jiang, B. (2018). Modular and Integral Knowledge Integration: From the Case of a Chinese IT Enterprise. Journal of Information \& Knowledge Management, 17(01), 1850012 .

Wynn, E., \& Novick, D. G. (1995, August). Conversational conventions and participation in cross-functional design teams. In Proceedings of the conference on Organizational computing systems (pp. 250-257). ACM. https://doi.org/10.1145/224019.224046

Yin, R. K. (2017). Case study research and applications: Design and methods. Sage publications. 


\section{Mll Macrothink}

International Journal of Human Resource Studies

ISSN 2162-3058 2018, Vol. 8, No. 3

\section{Appendix}

Appendix 1. A List of Acronyms in the Paper

\begin{tabular}{lc}
\hline & List of Acronyms \\
\hline KI & Knowledge Integration \\
CFT & Cross-Functional Team \\
MASI & Mobile Application Software Industry \\
SD & Standard Deviation \\
CTO & Chief Technology Officer \\
OLS & Ordinary Least Squares \\
H1 & Hypothesis 1 \\
H2a & Hypothesis 2a \\
H2b & Hypothesis 2b \\
H3a & Hypothesis 3a \\
H3b & Hypothesis 4b \\
\hline
\end{tabular}

\section{Copyright Disclaimer}

Copyright for this article is retained by the author(s), with first publication rights granted to the journal.

This is an open-access article distributed under the terms and conditions of the Creative Commons Attribution license (http://creativecommons.org/licenses/by/4.0/). 